\title{
In memoriam Prof. Dr. med. Dr. h. c. mult. Walter Frommhold, * 28. Aug. 1921, $\dagger$ 18. Sept. 2010
}

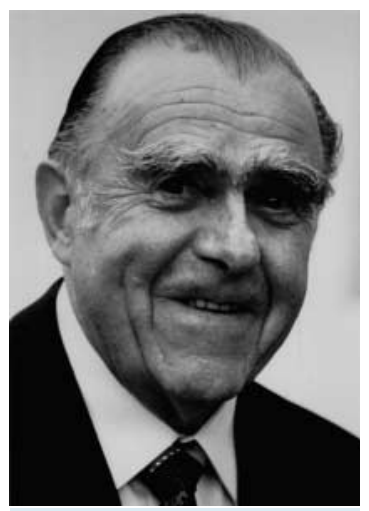

Prof. Dr. Walter Frommhold
Am 18. September 2010 starb Walter Frommhold, em. o.Prof. Dr. Dr. h.c. mult., ehemaliger Direktor des Medizinischen Strahleninstituts der Eberhard-Karls-Universität Tübingen im Alter von 89 Jahren.

Die deutsche Radiologie verlor mit ihm einen herausragenden Arzt und Vertreter unseres Faches, Frommhold zählte zu den prominentesten Radiologen Europas, er hatte maßgeblichen Anteil an der Gestaltung der deutschen Radiologie über viele Jahrzehnte hinweg. Stets war er eingebunden in eine Vielfalt beruflicher und persönlicher Freundschaften.

In Geringswalde/Sachsen geboren, begann er nach Volks- und Oberschule an der FriedrichWilhelms-Universität in Berlin das Studium der Medizin und legte 1944 das Staatsexamen in Würzburg ab.

Im Dezember 1946 begann er seine Facharztausbildung im Fach Röntgenologie und Strahlenheilkunde bei Prof. Dr. Schön am Zentralen Röntgeninstitut der Städtischen Krankenanstalten Karlsruhe. Nach der Facharztanerkennung wechselte er im April 1951 nach Berlin an das Strahleninstitut der Freien Universität und wurde Oberarzt bei Prof. Dr. H. Oeser. Dort erhielt er 1955 die Venia legendi der Freien-Universität Berlin für das Fach Röntgenologie und Strahlenheilkunde.

In diese Zeit fallen auch seine wissenschaftlichen Arbeiten über Kontrastmittelfragen, speziell der Gallenblasendiagnostik. Im Anschluss an diese Oberarztzeit wechselte er als Chefarzt an das Städtische Auguste-Viktoria-Krankenhaus Berlin-Schöneberg. Diese moderne, leistungsfähige, auf allen Gebieten der Radiologie tätige Abteilung erlangte unter seiner Leitung schnell einen über die Grenzen Berlins hinausreichenden Ruf, und schon damals drängte es viele jüngere Radiologen, ihre Weiterbildung in der „Schule Frommhold“ durchzuführen.

Nach einem Aufenthalt als „Teaching Fellow“ in der Abteilung für Radiologie im Harvard College in Boston USA folgte 1962 die Ernennung zum außerplanmäßigen Professor. Nach einem abgelehnten Ruf auf die ordentliche Lehrkanzel für Röntgenologie an der Universität Wien nahm er 1968 den Ruf als ordentlicher öffentlicher Professor für medizinische Strahlenkunde und Direktor des medizinischen Strahleninstituts der Universität Tübingen an.

Bibliografie

DOI http://dx.doi.org/10.1055/ s-0029-1245881

Fortschr Röntgenstr 2010; 182: 1065 ๑ Georg Thieme Verlag KC Stuttgart · New York · ISSN 14389029 leninstituts und das geistige Umfeld der altehrwürdigen Universität Tübingen bildeten die ideale Basis für das Wirken Walter Frommholds. Unter seiner Leitung wurde die Stellung der Tübinger Radiologie als eines der führenden Institute der Bundesrepublik ausgebaut. Das Medizinische Strahleninstitut Tübingen war eines der ersten in Deutschland, das die neuesten radiologischen Methoden wie Computertomografie, Magnetresonanztomografie oder in der Radioonkologie den Linearbeschleuniger anwandte.

Eine große Zahl wissenschaftlicher Publikationen auf sämtlichen Gebieten der Radiologie zeugen von herausragenden wissenschaftlichen Aktivitäten.

Während seiner Zeit als Vorsitzender der Deutschen Röntgengesellschaft 1971 - 1975 gelang es Walter Frommhold, das Bild der Radiologie als klinisches Fach bei anderen Disziplinen der Medizin zu festigen und auszubauen. Seine besondere Dynamik, Begeisterungsfähigkeit und Ausstrahlungskraft kennzeichnete sein Wirken in jeder seiner zahlreichen ärztlichen und standespolitischen Funktionen. Als Präsident des 4. Europäischen Kongresses für Radiologie in Hamburg war Frommhold Gastgeber für die Radiologen Europas.

Eine besondere Zuwendung galt immer der wissenschaftlich-publizistischen Tätigkeit, insbesondere in Zusammenarbeit mit dem Georg Thieme Verlag Stuttgart. So fanden enge persönliche und fachliche Beziehungen mit der Familie Hauff über mehrere Generationen hinweg ihren Niederschlag in der gesamten medizinischen Literatur. Nicht nur die Fachzeitschrift „RöFo - Fortschritte auf dem Gebiet der Röntgenstrahlen und der bildgebenden Verfahren“ standen für ihn im Zentrum seiner Herausgeberschaften, auch eine lange Reihe von radiologischen Lehrbüchern prägte die Radiologie weit über die Grenzen Deutschlands hinaus.

Es ist kaum möglich, in diesen kurzen Zeilen seinem Wirken, Wesen, seiner Dynamik und Durchsetzungskraft gerecht zu werden. Eine Vielzahl akademischer und gesellschaftlicher Ehrungen in nationalen und internationalen Gesellschaften und Universitäten legt davon Zeugnis ab. Seinen Schülern und Mitarbeitern war er als akademischer Lehrer und engagierter Arzt stets ein erstrebenswertes Vorbild. Neben allen bürokratischen und organisatorischen Herausforderungen blieb jedoch der anvertraute Patient stets Mittelpunkt und letzte Instanz seiner Entscheidungen. Wir ehren in Walter Frommhold einen Arzt und Menschen, der nicht nur unser Fach, sondern auch unser akademisches und berufliches Leben geprägt hat.
Für seine Schüler: Karl-Jürgen Wolf Für den Georg Thieme Verlag und die Herausgeberschaft: Albrecht Hauff 